\title{
Regulatory effect of heat shock transcription factor-1 gene on heat shock proteins and its transcriptional regulation analysis in small abalone Haliotis diversicolor
}

Xin Zhang ${ }^{1,2,3}$, Yuting $\mathrm{Li}^{3}$, Yulong Sun ${ }^{1}$, Mingxing Guo ${ }^{1}$, Jianjun Feng ${ }^{2,3}$, Yilei Wang ${ }^{2,3^{*}}$ and Ziping Zhang ${ }^{1,4^{*}}$ (D)

\begin{abstract}
Background: The effects of diverse stresses ultimately alter the structures and functions of proteins. As molecular chaperones, heat shock proteins (HSPs) are a group of highly conserved proteins that help in the refolding of misfolded proteins and the elimination of irreversibly damaged proteins. They are mediated by a family of transcription factors called heat shock factors (HSFs). The small abalone Haliotis diversicolor is a species naturally distributed along the southern coast of China. In this study, the expression of HdHSFI was inhibited by RNAi in hemocytes in order to further elucidate the regulatory roles of HdHSF1 on heat shock responsive genes in abalone. Meanwhile, to understand the transcriptional regulation of the HdHSF1 gene, the 5'-upstream regulatory region of HdHSF1 was characterized, and the relative promoter activity was examined by dual-luciferase reporter gene assay system in HEK293T cell lines.
\end{abstract}

Results: After the inhibition of the H. diversicolor HSF1 gene (HdHSF1) by dsRNA (double-stranded RNA), the expression of most heat shock related-genes was down-regulated $(p<0.05)$. It indicated the importance of HdHSF1 in the heat shock response of H. diversicolor. Meanwhile, $5^{\prime}$-flanking region sequence (2633 bp) of the HdHSF1 gene was cloned; it contained a putative core promoter region, TATA box, CAAT box, CpG island, and many transcription elements. In HEK293T cells, the $5^{\prime}$-flanking region sequence can drive expression of the enhanced green fluorescent protein (EGFP), proving its promoter function. Exposure of cells to the high-temperature $\left(39^{\circ} \mathrm{C}\right.$ and $\left.42^{\circ} \mathrm{C}\right)$ resulted in the activation of $H d H S F 1$ promoter activity, which may explain why the expression of the HdHSF1 gene participates in heat shock response. Luciferase activity of different recombinant plasmids, which contained different truncated promoter fragments of the HdHSF1 gene in HEK293T cells, revealed the possible active regions of the promoter. To further identify the binding site of the critical transcription factor in the region, an expression vector with the site-directed mutation was constructed. After being mutated on the GATA-1 binding site, we found that the luciferase activity was significantly increased, which suggested that the GATA-1 binding site has a certain weakening effect on the activity of the HdHSF1 promoter.

Conclusions: These findings suggest that GATA-1 may be one of the transcription factors of HdHSF1, and a possible signaling pathway mediated by HdHSF1 may exist in H. diversicolor to counteract the adverse effects of heat shock stress.

Keywords: Haliotis diversicolor, Heat shock transcription factor-1, RNAi, Transcriptional regulation, GATA-1

\footnotetext{
* Correspondence: ylwang@jmu.edu.cn; zhangziping@hotmail.com

${ }^{2}$ Fujian Engineering Research Center of Aquatic Breeding and Healthy Aquaculture, Xiamen 361021, China

${ }^{1}$ College of Animal Science, Fujian Agriculture and Forestry University, Fuzhou 350002, China

Full list of author information is available at the end of the article
}

C C The Author(s). 2020 Open Access This article is licensed under a Creative Commons Attribution 4.0 International License, which permits use, sharing, adaptation, distribution and reproduction in any medium or format, as long as you give appropriate credit to the original author(s) and the source, provide a link to the Creative Commons licence, and indicate if changes were made. The images or other third party material in this article are included in the article's Creative Commons licence, unless indicated otherwise in a credit line to the material. If material is not included in the article's Creative Commons licence and your intended use is not permitted by statutory regulation or exceeds the permitted use, you will need to obtain permission directly from the copyright holder. To view a copy of this licence, visit http://creativecommons.org/licenses/by/4.0/. The Creative Commons Public Domain Dedication waiver (http://creativecommons.org/publicdomain/zero/1.0/) applies to the data made available in this article, unless otherwise stated in a credit line to the data. 


\section{Background}

Different stresses (for example, exposure to high temperature, hypoxia, heavy metals, and bacterial infections) can affect the structure and function of proteins [1]. The accumulation of denatured and aberrantly folded proteins enhances the synthesis of heat shock proteins (HSPs) that are a group of highly conserved proteins. They act as molecular chaperones by helping in the refolding of misfolded proteins and assisting in the elimination of irreversibly damaged proteins [2, 3]. Exposure to a multitude of stressors can activate the cell's heat shock response (HSR). A family of transcription factors called heat shock factors (HSFs) bind to the heat shock elements (HSEs) that present in the promoter regions of HSP genes, mediates HSR and induces expression of HSPs [4]. Upon activation, each HSF undergoes extensive post-translational modifications and forms a transcriptionally active trimer that accumulates in the nucleus and acts on the target gene [5].

The HSF family consists of four different types: HSF1, HSF2, HSF3, and HSF4 [6]. HSF1, HSF2, and HSF4 had been identified in mammals, while HSF3 was described in chicken [7]. In vertebrates, HSF1 is thought to be the most important factor that induces thermal responses by regulating the refolding and assembly of HSPs, which are directly related to animal disease and life expectancy [8]. In invertebrates, HSF is required not only for the heat shock response but also for cell growth and differentiation and normal lifespan in yeast, Caenorhabditis elegans, and Drosophila [9-11]. HSF1 can drive the expression of a broad range of heat-responsive genes such as HSP90 in Drosophila during stress [12]. While several studies amply illustrate that HSP denaturation induces HSF1 expression, the exact molecular mechanisms about HSF1 transcriptional regulation remain unclear.

The small abalone Haliotis diversicolor is of great commercial value due to its unique nutrition and delicious taste [13]. However, the abalone industry has been severely affected by the frequent occurrence of infectious diseases and the deterioration of its environment, especially the hypoxia and thermal stress in hot summer months. These factors have threatened the abalone industry for a long time [14-17]. The high temperature in summer months along the southern coast can typically diminish the amount of dissolved oxygen, resulting in changes in metabolic and respiratory rates, and disease and high mortality of farmed abalones [16].

In the previous studies conducted by our team, several heat-shock related genes, such as heat-shock factor binding protein 1 (HSBP1), HSP90, and HSF1 have been cloned and characterized from $H$. diversicolor [15]. Some other heat-shock related genes, such as HSP22, HSP26, HSP60, HSP70, HSP105, and SIP, have also been demonstrated to be up-regulated by thermal stress in hemocyte and hepatopancreas [18]. Moreover, the transcriptional regulation of HdHSP90, HdHSC70, and HdHSP70 genes were all analyzed. The results indicated that HSEs were all presented in the 5 '-flanking sequence of the three HSP genes, which can also be bound by HSF [19-21].

In this study, to further elucidate the regulatory effect of HdHSF1 on other heat shock responsive genes in the small abalone, the expression of HdHSF1 was inhibited by RNAi in hemocytes, and then the expression of this gene and other genes was assessed by quantitative realtime PCR (qRT-PCR). Meanwhile, to understand the transcriptional regulation of the HdHSF1 gene, the $5^{\prime}$ upstream regulatory region of HdHSF1 was characterized, and the relative promoter activity of sequential deletion constructs and site-directed mutagenesis construct containing the vital cis-acting element was examined by dual-luciferase reporter gene assay system in HEK293T cell lines. The findings will provide new insights into the regulation of HSF1 expression and the mechanism of abalone to resist heat shock or other stresses.

\section{Results \\ Expression of HSR related genes when the HdHSF1 is inhibited by dsRNA}

Several heat-shock related genes have been reported to be regulated by thermal stress in our previous studies $[15,18]$. To further study the importance of the HdHSF1 gene on these HSR genes, dsRNA (double-stranded RNA) was used to inhibit the expression of the HdHSF1 gene. The expression of HdHSF1 was tested by qRT$\mathrm{PCR}$, and the results showed that the gene expression in the experimental group was significantly decreased $(p<$ 0.05) compared with the GFP RNAi group (control group) and the blank control group (Fig. $1 \mathrm{~A}$ ). After the inhibition of HdHSF1, the expressions of HSP22, HSP26, HSP60, HSP70, HSP90, HSP105, and HSBP1 were also significantly lower than those of the GFP RNAi group and blank control group (Fig. 1 B). However, the expression of SIP was not significantly affected by the interference of HdHSF1 ( $p>0.05)$.

\section{5' upstream sequences of HdHSF1 gene}

The 5 'flanking sequence (2633 bp) of the HdHSF1 gene was obtained by Tail-PCR and Genome Walker methods. The bioinformatics analysis showed that the predicted transcriptional start site (TSS) is located at $149 \mathrm{bp}$ upstream of the start codon (ATG), and the core promoter region is located at $-40 \mathrm{bp}$ to $+5 \mathrm{bp}$ when the TSS was specified as 1 . The predicted promoter region has a TATA box between - 26 and - 33 bp, a CAAT box between -82 and $-86 \mathrm{bp}$, a CpG island with a length of 189 bp ( 902 to -1090$)$, and many transcription factor 
binding sites such as GATA-1, NF-1, SRF, Sp1, Oct-1, CTF, C-JUN, and USF are included (Fig. 2).

\section{Activity analysis of $H d H S F 1$ promoter in vitro}

To further characterize the promoter functionality of the HdHSF1 gene, 2633 bp $5^{\prime}$-upstream region was inserted into the pEGFP-1 vector (pEGFP-hsf1) and used to drive the expression of the EGFP gene in HEK293T cells. The pEGFP-N1 promoter used as a positive control had high fluorescence activity as expected. No green fluorescence protein expression was detected in PEGFP-1 as a negative control (Fig. 3).

To identify the core promoter region of the HdHSF1 gene, two constructed reporter plasmids (one containing 1963 bp 5'-upstream region was named pGL3-hsf1-1r; the other one fragment removing the core promoter region was named pGL3-hsf1-1rr) were prepared and transfected into HEK293T cells. The activity of pGL3hsf1-1r was significantly higher than that of pGL3-hsf1$1 \mathrm{rr}$ and negative control (pGL3-Basic, plasmid without insert any target fragments) $(p<0.05)$ (Fig. 4).

To determine if the expression of the HdHSF1 gene promoter was induced by heat shock, after we transfected the pGL3-hsf-1r plasmid into HEK293T cells, the cells were incubated at $37^{\circ} \mathrm{C}, 39^{\circ} \mathrm{C}$, and $42{ }^{\circ} \mathrm{C}$ for 40 $\mathrm{min}$. The results showed that under the induction of $39^{\circ} \mathrm{C}$ and $42^{\circ} \mathrm{C}$, the luciferase activity was significantly increased $(p<0.05)$, and the activity in $39^{\circ} \mathrm{C}$ was the highest (Fig. 5).

To identify important transcription factor binding sites in the HdHSF1 promoter region, we transferred a series of different spans of the predicted promoter region containing the transcriptional factor binding element into pGL3-Basic luciferase report vector respectively (named as pGL3-hsf1-r1, pGL3-hsf1-r2, pGL3-hsf1-r3, pGL3-hsf1-r4, pGL3-hsf1-r5, pGL3hsf1-r6, and pGL3-hsf1-r7). The constructs were used to transfect into HEK293T cells. The results showed that all truncated promoters had detectable activities compared with control (pGL3-Basic, plasmid without insert any target fragments). There were significant differences between pGL3-hsf1-r3 and pGL3-hsf1-r4 or between pGL3-hsf1-r6 and pGL3-hsf1-r7 $(p<0.05)$ (Fig. 6).

The difference between pGL3-hsf1-r3 and pGL3-hsf1$\mathrm{r} 4$ is the part of -1108 to $-935 \mathrm{bp}$. There are many predicted transcription factors binding sites in this region, such as Oct-1, GATA-1, Sp1, SRF, NF-1, USF, etc. The TG of the binding site of the transcription factor GATA-1 (ATCTGTTCCC) was mutated into CA (ATCCATTCCC), and the mutant recombinant plasmid was named as pGL3-mut-ga. The results showed that after the gata- 1 binding site was mutated, the luciferase activity significantly increased $(p<0.05)$ (Fig. 7).

\section{Discussion}

Diverse stresses, e.g. exposure to heat shock, heavy metal ions, hypoxia, and bacterial infection, have been known to cause denaturation and aggregation of proteins, to disrupt the integrity of essential organelles, and to inhibit vital processes, such as transcription and mRNA translation [1, 22, 23]. The cell response to proteotoxic stresses is mediated primarily through the activation of HSF1 [24]. HSF activates transcription in response to cellular stress. Human HSF1 was proved to contain a central regulatory domain that can repress the activity of its activation domains [25]. A highly conserved DNAbinding domain that can be regulated by interactions between the transcriptional activation domain and the amino-terminal negative regulator might have similar functions in vertebrates and invertebrates [26-29].

Furthermore, the constitutive serine phosphorylation sites were also be proved to have a central role in the negative regulation of HSF1 transcriptional activity by transfected mammalian cells [30]. In the previous studies, the full-length cDNA sequences of HdHSF1 were cloned successfully [15]. The result of the sequence analysis showed that HdHSF1 also contained a heat shock factor domain [15], which may be consistent with the functional descriptions in humans [25]. Serine phosphorylation sites that have been reported to have an essential role in the negative regulation of HSF1 transcriptional activity in mammalian cells [30] were also found in HdHSF1 [15].

The expression level of HdHSF1 was also demonstrated that it would significantly be up-regulated in gills and hemocytes after heat shock or hypoxia stress to protect cells from damage [15]. It indicated that HdHSF1 might be involved in the regulation of heat shock response in abalone [15]. Otherwise, HSEs which could be bound by HSFs to mediate HSR and the induction of HSPs were found in the 5 'flanking sequence of HdHSC7O [20], HdHSP7O [19], and HdHSP9O [21] and it indicated that they may all be regulated by HdHSF1. So far, the function and regulation of HSF1 in Mollusca are very limited. This study provides a theoretical basis to HSF1 regulation mechanisms by cloning, bioinformatics analysis, the transcriptional activity of the $5^{\prime}$-flanking region of $H d H S F 1$, and identifying the critical elements involved in its regulation.

\section{The expression of HSR genes after the inhibition of HdHSF 1}

RNA interference has been proved to be an effective method to study the interaction of different genes. Nowadays, with the rapid development of molecular biological techniques, dsRNA interference has been successfully carried out in Biomphalaria glabrata [31], 


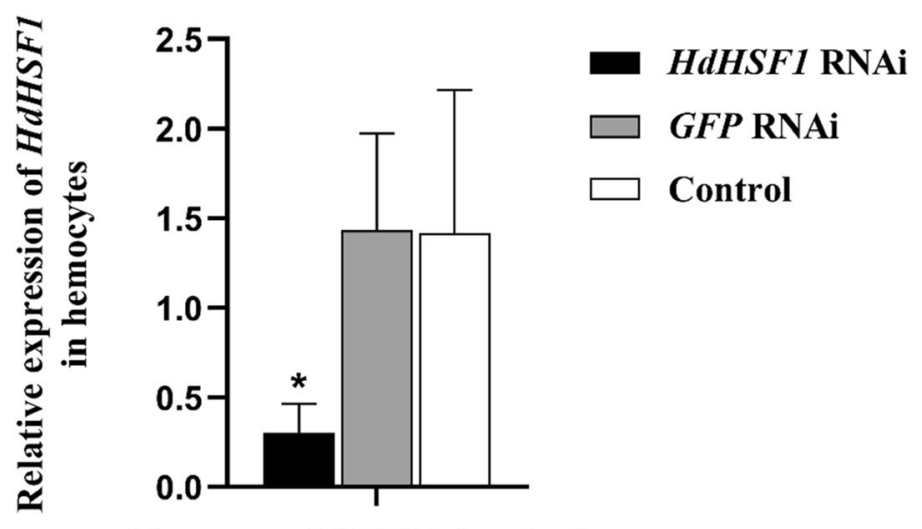

A

6 hours post dsRNA incubation
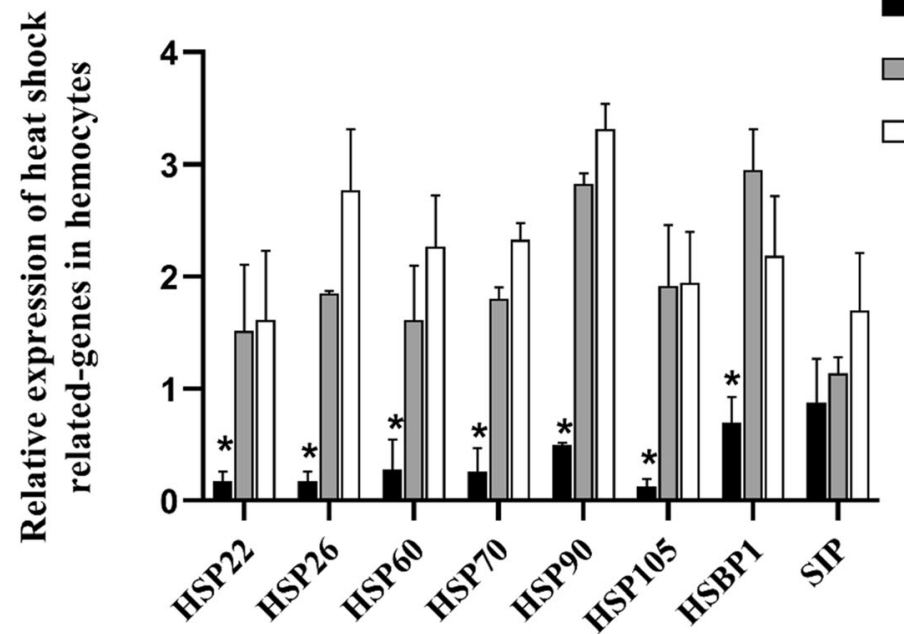

- HdHSF1 RNAi

$\square$ GFP RNAi

\section{Control}

B

\section{6 hours post dsRNA incubation}

Fig. 1 Expression analysis of the heat shock-related genes when the HdHSF1 was inhibited by dsRNA in hemocytes. a. the mRNA expression level of the HdHSF1 gene in the HdHSF1 RNAi group was significantly downregulated compared with the GFP RNAi group and the blank control group $(p<0.05)$. b. the mRNA expression levels of 8 heat shock-related genes after the interference of HdHSF7. The X-axis represents treatment conditions and different target heat shock responsive transcripts. Y-axis represents the mRNA expression level of different genes. Six biological replicates were tested, and each sample was assayed in triplication. A significant difference between the experimental group and the control group was indicated by a $\left(^{*}\right)$ at $p<0.05$. Control: blank control group. GFP-RNAi: group in which green fluorescent protein (GFP) gene was inhibited by dsRNA. HdHSF1-RNAi: group in which HdHSF1 was inhibited by dsRNA

and $H$. diversicolor in our previous study [32-34]. RNAi is initiated by the enzyme Dicer, which cleaves long dsRNA molecules into short double-stranded siRNAs. The well-studied outcome is post-transcriptional gene silencing. The activated RISC-siRNA complex scans, binds, and degrades the complementary target mRNA and leads to gene silencing [35].

To understand the regulation of HdHSF1 on other genes associated with heat shock in $H$. diversicolor, the HdHSF1 was transcriptionally inhibited by dsRNA in hemocytes in this study. The qRT-PCR result showed that the expression of HdHSF1 in the experimental group was significantly lower than the GFP RNAi group and the blank control group, indicating $H d H S F 1$ was knocked down successfully. After the inhibition of HdHSF1, the expression of HSP22, HSP26, HSP60, HSP70, HSP90, HSP105, and HSBP1 was down-regulated. This result also indicated that HdHSF1 had a positive regulatory effect on these genes. Although the expression of SIP was significantly up-regulated by thermal stress [18], no significant decrease of SIP in response to HdHSF1 silencing indicated that it might be regulated by other factors and had no relation with the HdHSF1 gene. 


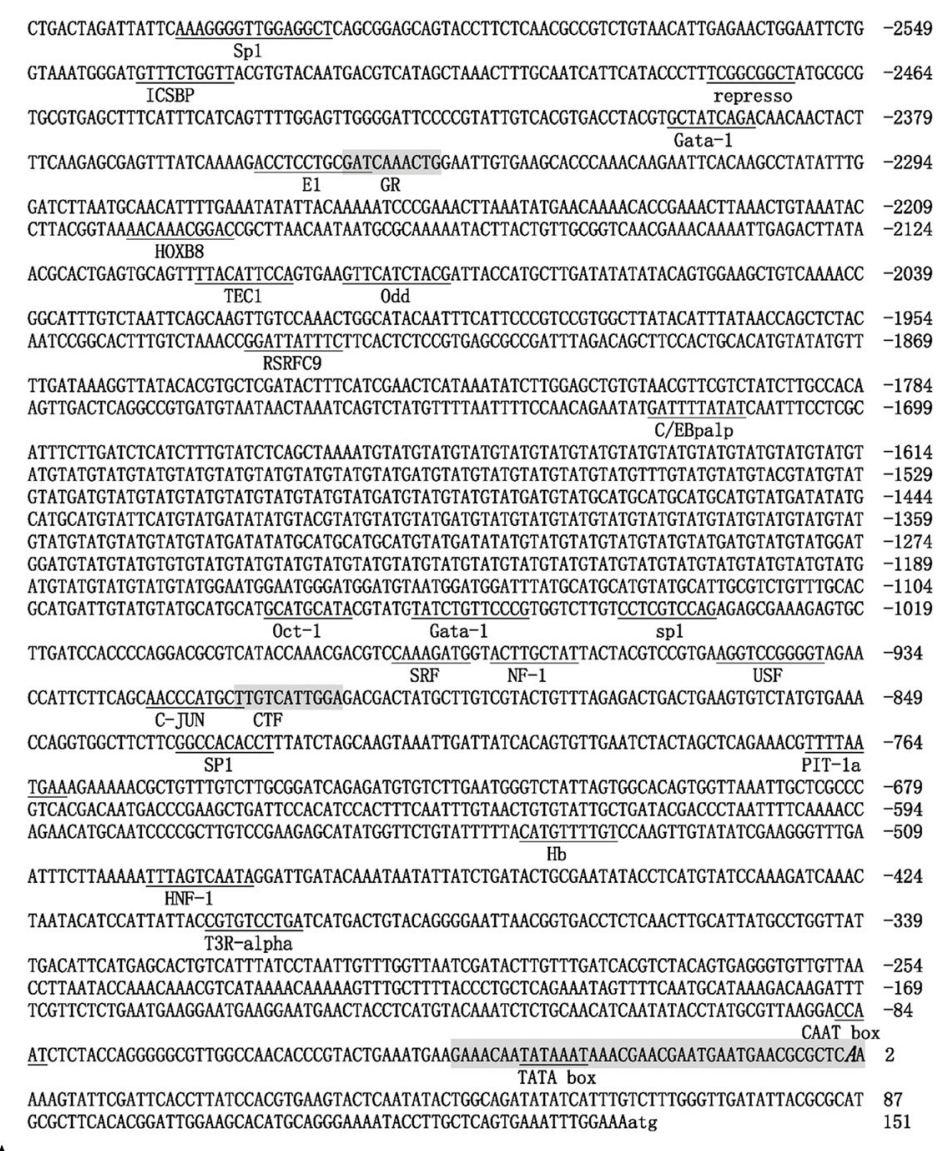

A

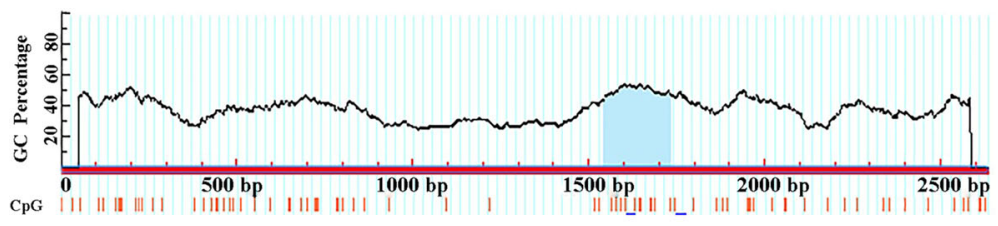

B

Fig. 2 The nucleotide sequence of the $5^{\prime}$-flanking region of HAHSF1. a. The potential binding sites of the transcription factors are marked with a short, thin line. Overlapping binding sites are indicated by shading. The predicted core promoter region is shaded, the transcription start site in a bold and italic letter, and is located at 1, and the translation start site (atg) is bolded and lowercase. b. CpG islands (blue shadow) in the 5'-flanking sequence of HdHSF1 gene

\section{The HdHSF1 5' upstream sequence}

The predicted result by the bioinformatics analysis showed that $\mathrm{CpG}$ island, which has been proved to be involved in regulating gene expression, was contained in the 5 '-flanking sequence $[36,37]$. The predicted result showed that the CpG island of HdHSF1 was far from the TSS, which was similar to the result of the HdHSC7O gene but different from that of the HdHSP70 gene in $\mathrm{H}$. diversicolor $[19,20]$. The typical CpG islands of eukaryotic genes which can initiate transcription were near or appear on the TSS [38]. Furthermore, the CpG island, which was far from the annotated TSS, has also been indicated to have promoter-like characteristics and was involved in the transcriptional regulation of genes [39].
The TATA box is one of the components in a eukaryotic promoter, which is the most critical binding site of eukaryotic RNA polymerase II, and the sequence pattern is TATAATAAT [40]. Early studies suggested that the TATA box was necessary for the correct transcription of all eukaryotic structural genes. With the development of large-scale genome sequencing, more and more eukaryotic gene sequences were identified, and it was found that there was no TATA box in the $5^{\prime}$ flanks of many genes and elements such as the downstream promoter element (DPE) and the initiator (Inr) could also bound to TFIID in the transcription of core promoters in the absence of a TATA box [41]. In this study, a TATA box was found to be located at $181 \mathrm{bp}$ 

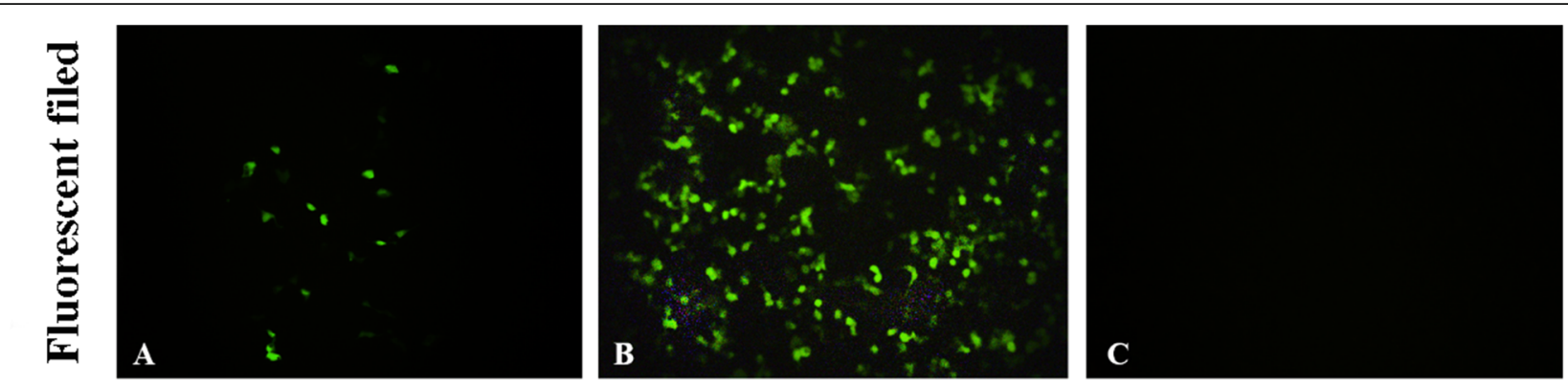

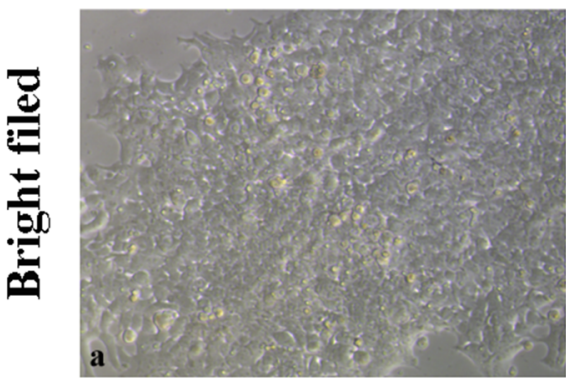

pEGFP-hsf1

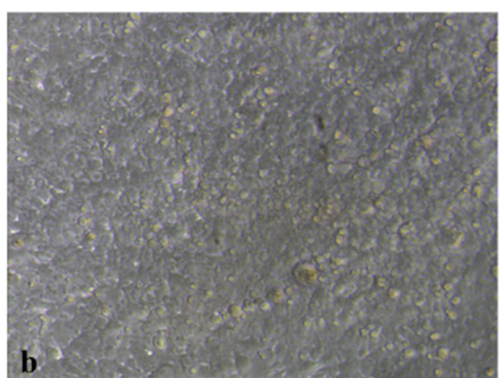

pEGFP-N1

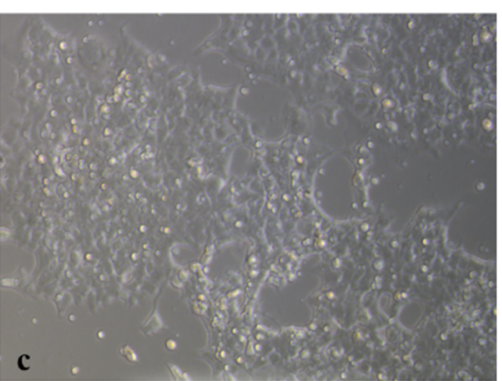

pEGFP-1

Fig. 3 The expression of pEGFP-hsf1 in HEK293FT cells. The EGFP expression of the HdHSF1 promoter in HEK293T cells at $24 \mathrm{~h}$ post transfection with pEGFP-hsf1, which used the HdHSF1 full-length promoter (A and $\mathbf{a})$, pEGFP-N1 as a positive control (B and $\mathbf{b})$ and promoter-less pEGFP-1 as a negative control (C and $\mathbf{c})$. Fluorescent fields are shown in $(\mathbf{A}, \mathbf{B}$, and $\mathbf{C})$, and bright fields are observed in (a, b, and $\mathbf{c})$ separately

upstream of initiation codon ATG in the core promoter region of $H$. diversicolor and the loss of this region led to a significant decrease in the activity of the promoter, indicating that HdHSF1 gene expression was regulated by TATA box.

Regulatory elements are needed for a promoter to sustain transcription in vivo. Transcription factors have to bind to the cis-acting elements to start transcription, no matter they are activators or repressors [42]. Due to the lack of a stable cell line of $H$. diversicolor, the HEK293T cell line, which has been widely used in vertebrate and invertebrate promoter functional analysis [19, 20, 43, 44], was used for the promoter assay in this study. The detection of the promoter activity and the determination

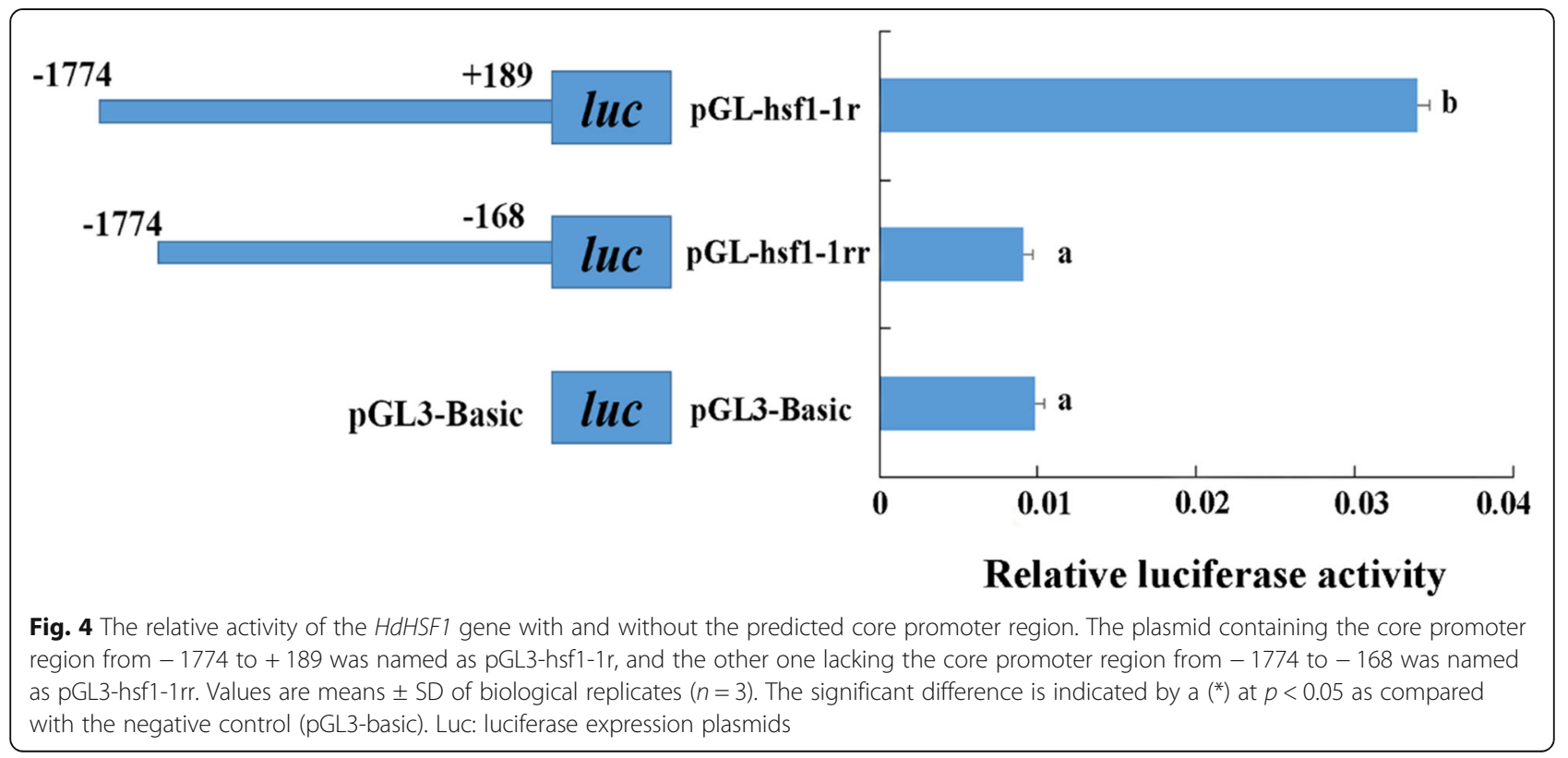




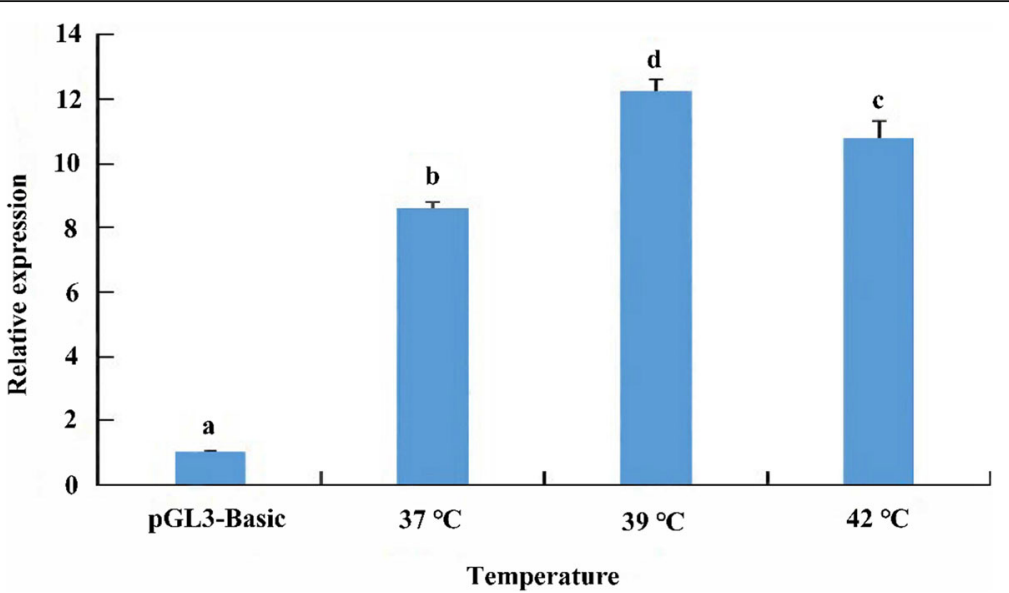

Fig. 5 Changes of HdHSF1 promoter activity in HEK293T cells under high temperature. The cells were incubated at different temperatures $\left(37^{\circ} \mathrm{C}\right.$, $39^{\circ} \mathrm{C}$, and $42^{\circ} \mathrm{C}$ ) for 40 mins. The means \pm SD of biological replicates $(n=3)$ were used to present the relative expression. The pGL3-basic plasmid served as a negative control. The different letters on the error bars represent significant differences, $p<0.05$

of the transcription initiation site were carried out in this study to further characterize the function of the HdHSF1 promoter. The activity of the complete $2633 \mathrm{bp}$ promoter of $H d H S F 1$ was verified using the fluorescent expression on the transfected cells with the promoterEGFP vector (Fig. 3). The luciferase activity decreased significantly in pGL3-hsf1-1rr compared to pGL3-hsf1$1 \mathrm{r}$ (Fig. 4) suggested that the core promoter region of the HdHSF1 gene was located between - $168-+189 \mathrm{bp}$.
HSFs can induce the expression of HSPs by binding to the HSEs present in the promoter regions of HSP genes. So far, the researches on the promoter of the HSFs were limited compared to that of HSPs. Previous results indicated that the HSP genes had an inducible promoter, and the transcription level of these genes significantly increased under high temperatures or other stresses [19, $20,45]$. In this study, the transfected cells were exposed to different temperatures to identify whether the activity

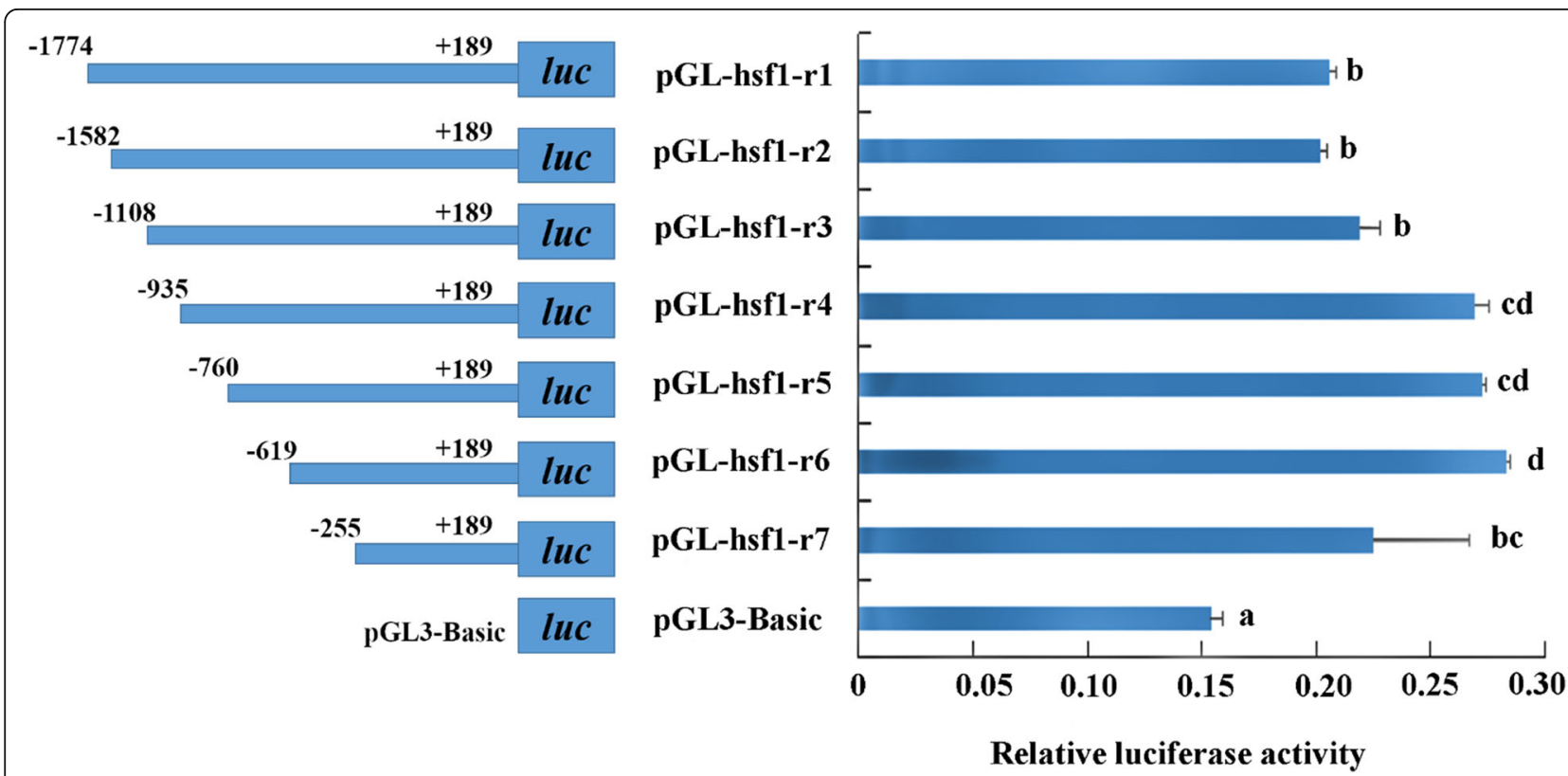

Fig. 6 Activity analysis of HdHSF1 gene promoter in HEK293T cells. Based on the length of the seven fragments containing promoter region, the recombinant plasmids were named pGL3-hsf1-r1, pGL3-hsf1-r2, pGL3-hsf1-r3, pGL3-hsf1-r4, pGL3-hsf1-r5, pGL3-hsf1-r6, and pGL3-hsf1-r7. The pRLTK vector containing the Renilla luciferase gene was transfected as an internal reference to correct the transfection efficiency. The pGL3-Basic plasmid served as a negative control. The different letters on the error bars represent significant differences, $p<0.05$. The values are means \pm SD of biological replicates $(n=3)$ 


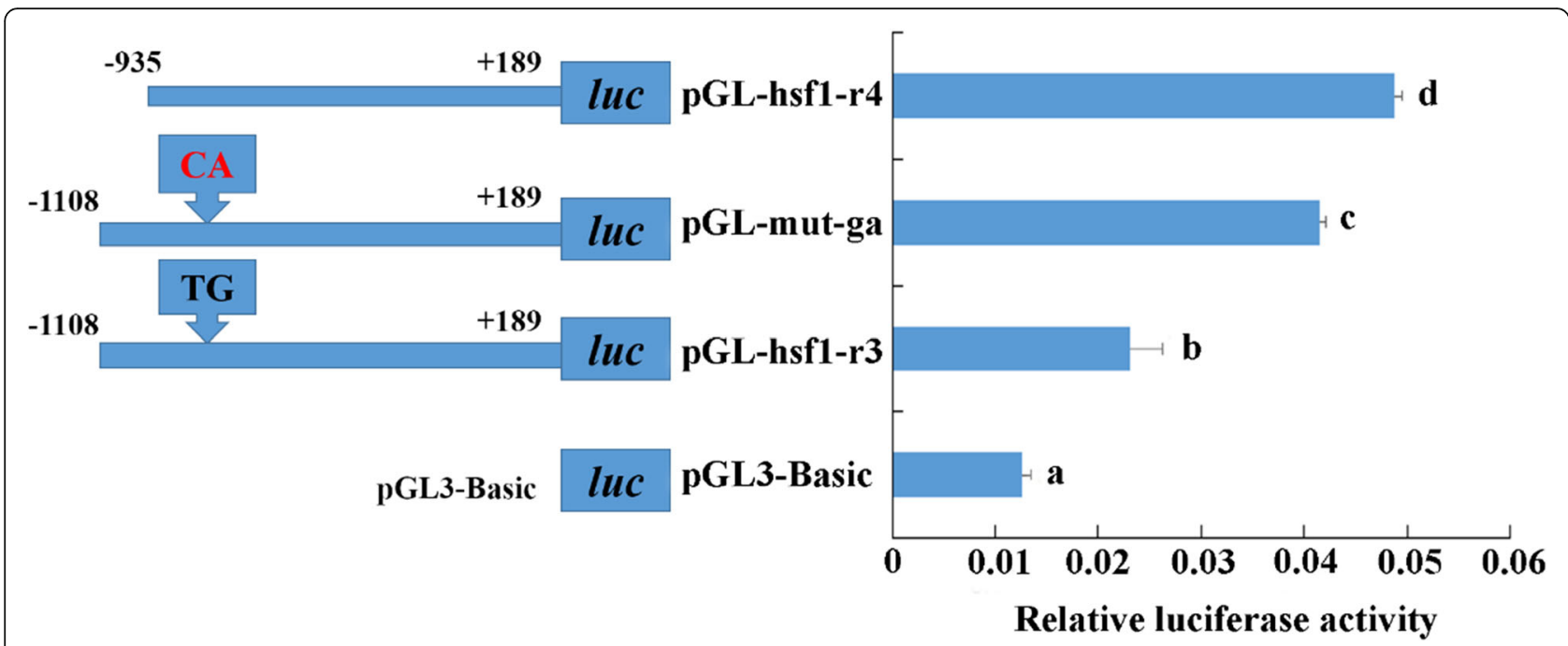

Fig. 7 Luciferase activity of the site-directed mutation plasmid pGL3-mut-ga. The distance between pGL3-hsf1-r3 and pGL3-hsf1-r4 contains a sole GATA-1 binding site (ATCTGTTCCC) in the promoter of HdHSF1. When TG was mutated into CA, the sequence became ATCCATTCCC, and the mutant recombinant plasmid was named pGL3-mut-ga. The pGL3-basic plasmid was served as a negative control. (The different letters on the error bars represent significant differences, $p<0.05)$. Luc: luciferase expression plasmids

of the HdHSF1 gene promoter was induced by heat shock. The result showed that the luciferase activity of HdHSF1 had a significant increase after the treatment of HEK293T cells at $39^{\circ} \mathrm{C}$ and $42^{\circ} \mathrm{C}$. It indicated that the activity of the HdHSF1 promoter could be regulated by thermal stress, which is the same as the expression pattern of the HdHSF1 mRNA in $H$. diversicolor under thermal stress [15]. Nevertheless, although the different high temperatures would cause a change of significant increase in luciferase activity of $H d H S F 1$, the activity of HdHSF 1 at $42{ }^{\circ} \mathrm{C}$ was lower than that at $39^{\circ} \mathrm{C}$. It indicated that excessive temperature would decrease the activity of the $H d H S F 1$ promoter, which was similar to the findings in humans [46].

By binding to the binding sites in the upstream region of genes, the positive or negative regulatory transcription factors could regulate the expression of genes [20]. The result of this study showed that all truncated promoters had detectable activities, while a significant difference appeared between pGL3-hsf1-r3 and pGL3-hsf1-r4 $(p<0.05)$. It indicated that a critical transcription factor existed in the deleted site $(-1108$ to $-935 \mathrm{bp}$ ), and it played a central role in the basic transcription of the HdHSF1 promoter. After mutation in the transcription factor binding site GATA-1 between pGL3-hsf1-r3 and pGL3-hsf1-r4, a certain enhancement effect on the activity of $H d H S F 1$ promoter was found. Thus, GATA factor may be a negative regulator for HdHSF1.

GATA factors are a family of transcription factors that contain a zinc finger. They can recognize the sequence $(\mathrm{A} / \mathrm{T}) \mathrm{GATA}(\mathrm{A} / \mathrm{G})$ and are involved in the regulation of gene expression and differentiation [47]. GATA factors have been identified in vertebrates, D. melanogaster, Caenorhabditis elegans, and plants [47, 48]. The previous study in HL-60 cells demonstrated that the fusion

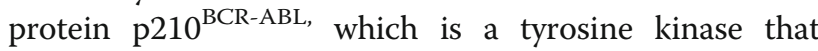
causes transformation and chemotherapy resistance, induces HSP-70 through GATA-1, a trans-factor that binds GATA response element at upstream of HSP-70 promoter [49]. The promoter activity of the fragment with GATA-1 binding sites deletion was significantly decreased. It revealed that GATA-1 could negatively regulate the transcription of the HdHSF1 gene. However, further research is necessary to clarify the specific regulation mechanism of GATA-1 on HdHSF1.

In summary, we demonstrated that HdHSF1 had a positive regulatory effect on other heat shock responsive genes in the small abalone. We cloned and characterized the promoter region of the small abalone HdHSF1 gene, discovered that GATA-1 was crucial for the transcriptional regulation of the HdHSF1 gene. It's the first time to analyze the promoter activity of the HSF1 gene in Mollusca, and the data might be helpful in further investigate the molecular mechanism of the specific expression pattern of the HSF1 gene and its regulation on other HSPs to assist in the elimination of irreversibly damaged proteins to resist heat shock or other stresses.

\section{Conclusions}

GATA-1 may be one of the essential transcription factors, which regulate the expression of the HdHSF1 gene. The inhibition of HdHSF1 induced the down- 
regulation of the other $H S P$ genes indicated that HdHSF1 had a positive regulatory effect on these genes. These results suggested that such a possible signal transduction pathway which the transcription factor GATA-1 could regulate the expression of HSF1 gene and then induced the expression of HSPs (except $S I P)$ to assisting in the elimination of irreversibly damaged proteins to resist heat shock or other stresses was existed in $H$. diversicolor.

\section{Methods}

\section{Animals and ethics statement}

Adult small abalones (body length $5.88 \pm 0.80 \mathrm{~cm}$, weight $16.7 \pm 1.80 \mathrm{~g}$ ) were purchased from the Peiyang abalone farm (Xiamen, Fujian Province). All these abalones were maintained in recycling systems with sand-filtered seawater at a temperature of $25^{\circ} \mathrm{C}$ and dissolved oxygen (DO) of $6.2 \mathrm{mg} / \mathrm{L}$ as described previously [14-16, 33, 50]. They were fed with sea tangle once a day and held before the experiment. All of the study design and animal experiments were conducted in accordance with the guidelines of Fujian Agriculture and Forestry University's Animal Care and Use Committee.

\section{Double-stranded RNA (dsRNA) preparation and exposure assay}

To elucidate the regulatory mechanisms of HdHSF1 on the other heat shock genes, RNA interference was performed by using the dsRNA of HdHSF1. The fragment of $H d H S F 1$ (The full-length cDNA of $H d H S F 1$ was registered in GenBank with accession No. KC688315) was amplified by PCR using gene-specific primers. The fragment of the GFP gene from the pEGFP-N1 vector was amplified by PCR. The sequences of two pairs of primers were shown in Additional file 1: Table S1. Singlestranded RNA (ssRNA) was transcribed from these PCR products by using T7 phage RNA polymerases (Promega, Shanghai, China). Then DNase I (Promega, Shanghai, China) was used to remove the trace amount of DNA at a ratio of $1 \mathrm{U} / \mu \mathrm{g}$. After being purified, the sense ssRNA and antisense ssRNA were mixed and annealed at $75^{\circ} \mathrm{C}$ for $15 \mathrm{~min}$, at $65^{\circ} \mathrm{C}$ for $15 \mathrm{~min}$, and then cool down to the room temperature at the rate of $0.2^{\circ} \mathrm{C} / \mathrm{s}$. The formation of dsRNA was monitored by checking the size shift in agarose gel electrophoresis, and the concentration of dsRNA was measured by using a spectrophotometer (NanoDrop ND-1000, Thermo Scientific, Shanghai, China).

The dsRNA of HdHSF1 was used in the silence experiment. Hemocytes were separately collected by cutting off the foot and were cultured in DMEM medium containing Penicillin-Streptomycin. Then, the hemocytes were divided into three groups:1) Experiment group: HdHSF1 dsRNA was added directly at a final concentration of $5 \mu \mathrm{g} / \mathrm{ml}$ to the hemocytes culture medium without any vehicle [51]. 2) The control group (GFP RNAi group): GFP dsRNA was added at a final concentration of $5 \mu \mathrm{g} / \mathrm{ml}$. 3) Blank control group: the medium without any modifications was regarded. There were six replicate beakers of each treatment group, and all samples were incubated at $27^{\circ} \mathrm{C}$ for $6 \mathrm{~h}$, and then the hemocytes were harvested to detect the mRNA expression by qRT-PCR.

\section{Isolation of total RNA and reverse transcription and qRT- $\mathrm{PCR}$ verification}

Total RNA was extracted by using total RNA Kit II (Omega, Shanghai, China) according to the manufacturer's protocol. The quality of total RNA was checked by electrophoresis and NanoDrop ND-1000. The cDNA was synthesized in a system including $1 \mu \mathrm{g}$ total RNA and $2 \mu \mathrm{L} 10 \mathrm{mM}$ random primers by M-MLV reverse transcriptase (Promega, Shanghai, China). The synthesized cDNA was diluted by 100 -fold and then stored at $-20^{\circ} \mathrm{C}$ until use.

Gene-specific primers for which we want to assay the expression level in RNAi experiment (Additional file 1: Table S1) were used to amplify products of 200-300 bp from cDNA, and the housekeeping $\beta$-actin gene of $H$. diversicolor (Accession No. AY436644) was selected as the reference gene $[14,16,33,52]$. qRT-PCR was carried out in a LightCycler480 Roche Real-time Thermal Cycler following the manual with a $10 \mu \mathrm{L}$ reaction volume containing $4.5 \mu \mathrm{L}$ of $1: 100$ diluted original cDNA, $5 \mu \mathrm{L}$ of $10 \times$ SYBR Green Master Mix (Promega, USA), and $0.25 \mu \mathrm{L}$ of each primer $(10 \mathrm{mM})$. The cycling conditions for the PCR reaction were set as follows: $1 \mathrm{~min}$ at $95^{\circ} \mathrm{C}$, followed by 40 cycles at $95^{\circ} \mathrm{C}$ for $15 \mathrm{~s}, 60^{\circ} \mathrm{C}$ for $1 \mathrm{~min}$. Melting curves were also plotted to ensure that a single PCR product was amplified for each pair of primers. The comparative $\mathrm{CT}$ method $(\Delta \mathrm{CT}=\mathrm{CT}$ of target gene minus CT of $\beta$-actin gene and $\Delta \Delta \mathrm{CT}=\Delta \mathrm{CT}$ of any sample minus calibrator sample) for the relative quantification of gene expression was used to calculate the relative expression level of all these genes. Six biological replicates were tested, and each sample was assayed in triplication. The t-test was used to determine the difference in the mean values among the treatments. The difference was considered significant when $p<0.05$.

\section{Cloning of the 5'-flanking regions of the HdHSF1 gene and bioinformatics analysis}

The $5^{\prime}$-flanking region of the HdHSF1 gene was obtained using the Tail-PCR and Genome Walker. The primer sequences used in this study are listed in Additional file 1: Table S1. PCR products were purified and cloned into the pMD19- $\mathrm{T}$ simple vector (TaKaRa, Dalian, China), and then sent to Sangon (Shanghai, China) for sequencing. 
The putative core promoter region and transcriptional start site (TSS) were predicted using online software, the Neural Network Promoter Prediction (NNPP) (http:// www.fruitfly.org/seq tools/promoter.html). The potential important transcription factor binding sites were analyzed by using the AliBaba2.1 (http://www.gene-regulation.com/pub/programs/alibaba2/index.html) database. The CpG islands were predicted by applying the MethPrimer with default parameters (http://www.urogene. org/cgi-bin/methprimer/methprimer.cgi).

\section{Cell culture, transfection and luciferase assays}

The HEK293T cells, obtained from the Eye Institute, Xiamen University, Xiamen, China, were routinely cultured in DMEM high glucose medium supplemented with $8 \%$ fetal bovine serum (FBS), $1 \%$ penicillin-streptomycin and grew at $37^{\circ} \mathrm{C}, 5 \% \mathrm{CO}_{2}$. Transfection experiments were performed in 48-well culture plates. One day before transfection, recipient cells were seeded into wells at a density of $1-3 \times 10^{5}$ cells/well. After removal of culture medium, the cells were transfected with $1 \mu \mathrm{g}$ of the reporter construct DNA and $0.02 \mu \mathrm{g}$ of internal reference plasmid in $50 \mu \mathrm{L}$ Opti-MEM medium per well using $1 \mu \mathrm{L}$ Lipofectin 2000 (Invitrogen, Shanghai, China) according to the manufacturer's recommendations. At $24 \mathrm{~h}$ posttransfection, the expression of enhanced green fluorescent protein (EGFP) was observed using an inverted fluorescence microscope.

After transfection, the culture medium was discarded, and the cells were washed one to two times in PBS. Each cell sample was then lysed by suspending in $60 \mu \mathrm{L}$ of $1 \times$ Passive Lysis Buffer (PLB). After centrifugation at $10000 \mathrm{~g}$ for $10 \mathrm{~min}$ at $4{ }^{\circ} \mathrm{C}$, the supernatant of each sample was taken as $15 \mu \mathrm{L}$. The reporter vectors pGL3-Basic (containing a firefly luciferase gene) and pRL-TK (containing a renilla luciferase gene) were obtained from Promega. The activity of firefly luciferase and luciferase of the plasmid were respectively recorded. The luciferase assay was performed using Dual-Glo luciferase assay system (Promega, USA) with pRL-TK vector (expressing Renilla luciferase under herpes simplex virus thymidine kinase promoter) employed as an internal control for normalization of transfection efficiency. The ratio of luciferase activity and the luciferase relative activity was calculated. All the data were obtained from three independent transfection experiments performed in triplicate.

\section{Construction and transient transfection of the EGFP plasmid}

Based on the 5 '-flanking region, the most extended 5 '-flanking DNA fragment was amplified from the genomic DNA of the $H$. diversicolor. The PCR product was cloned into a pMD19-T vector (TaKaRa, Dalian, China), and then double-digested with KpnI/ XhoI enzymes (TaKaRa, Dalian, China) and ligated to pEGFP-1, a promoterless EGFP report vector. The 5' flanking DNA fragment was located upstream of the EGFP gene. The recombinant vector was named as pEGFP-HSF1. Promoter activity of the $5^{\prime}$-flanking region was then tested by transfecting recombinant plasmid pEGFP-HSF1 into HEK293T cells, the pEGFP-1 and pEGFP-N1 plasmids were served as the negative and positive controls separately. After continuing culture for $24 \mathrm{~h}$, the cells were observed under a fluorescent microscope (Leica Microsystems, Wetzlar, Germany).

\section{Generation of reporter plasmid constructs}

To investigate whether the HdHSF1 promoter-driven luciferase reporter gene is induced by heat shock, HEK293FT cells were exposed at high temperatures of $37^{\circ} \mathrm{C}, 39^{\circ} \mathrm{C}$ and $42^{\circ} \mathrm{C}$ for 40 mins and then their luciferase activities were detected. To produce the luciferase reporter constructs including HdHSF1 5'-flanking DNA fragments with different lengths, multiple promoter fragments of the HdHSF1 gene were generated by PCR and cloned into the pGL3-Basic luciferase reporter vector. Firstly, the universal reverse primers were used in combination with different forward specific primers to create DNA fragments with different lengths and cloned into a pMD19-T simple vector (TaKaRa, Dalian, China). Secondly, the promoter fragment constructs were digested with Kpn I and Xho I, and sub-cloned into Kpn I/Xho Icut pGL3-Basic reporter vector. Finally, all plasmid constructs were verified by sequencing and purified with an E.Z.N.A. ${ }^{\text {Tu }}$ Endo-free Plasmid Mini Kit (OMEGA, Shanghai, China) for transfection.

Site-directed mutagenesis (SDM) was a PCR-based approach that can be used to identify the possible function of a specific cis-acting element with primers containing the mutational bases as well as the KpnI and XhoI restriction sites at each of the $5^{\prime}$-terminal separately. It was carried out by overlap extension PCR reactions with similar conditions and procedures, as mentioned above. After determining the transcription factor that may play an essential role in the regulation of gene expression, the interesting fragment with mutagenized cis-acting element was amplified by PCR then purified using a Wizard ${ }^{\circ}$ SV Gel and PCR Clean-Up System (Promega, USA) and inserted into pGL3-Basic vector containing the recombination sites upstream of the coding sequence of the firefly luciferase. The luciferase plasmid was then constructed. The following program was described as above. 


\section{Supplementary Information}

Supplementary information accompanies this paper at https://doi.org/10. 1186/s12860-020-00323-9.

Additional file 1: Table S1. Primers used in this article.

\section{Acknowledgements}

Not Applicable.

\section{Authors' contributions}

WYL and ZZP conceived the study and designed the experiments. ZX conducted the experiments and wrote the manuscript. LYT analyzed the data. SYL and GMX conducted the experiments. FJJ designed the experiments. WYL and ZZP checked and modified the manuscript. All authors read and approved the final manuscript.

\section{Funding}

This research was funded by the National Key R\&D Program of China (Grant Number: 2018YFD0900304-5) for Prof. Ziping Zhang who conceived the study and designed the experiments, checked and modified the manuscript; Discipline Development Grant from College of Animal Sciences FAFU (712018R0404) for Mr. Xin Zhang who conducted the experiments and wrote the manuscript, Open fund project of Fujian Engineering Research Center of Aquatic Breeding and Healthy Aquaculture (DF20902) for Mr. Xin Zhang; the Natural Science Foundation of China (No. 41176152) for Prof. Yilei Wang who conceived the study and designed the experiments, and checked and modified the manuscript; Special Fund for Marine Economic Development of Fujian Province (Grant Number: ZHHY-2019-3) for Prof. Ziping Zhang, International Science and Technology Cooperation and Communication Grant of Fujian Agriculture and Forestry University (Grant Number: KXGH17019) for Prof. Ziping Zhang, and 13th Five-year Plan on Fuzhou Marine Economic Innovation and Development Demonstration City Project (FZHJ17) for Prof. Ziping Zhang.

\section{Availability of data and materials}

We confirm that the materials described in the manuscript, including all relevant raw data, will be freely available to any scientist wishing to use them for non-commercial purposes, without breaching participant confidentiality.

\section{Ethics approval and consent to participate}

All of the study design and animal experiments were conducted in accordance with the guidelines of Fujian Agriculture and Forestry University's Animal Care and Use Committee.

\section{Consent for publication}

Not Applicable.

\section{Competing interests}

We have read and understood BMC policy on declaration of interests and declare that we have no competing interests.

\section{Author details}

${ }^{1}$ College of Animal Science, Fujian Agriculture and Forestry University, Fuzhou 350002, China. ${ }^{2}$ Fujian Engineering Research Center of Aquatic Breeding and Healthy Aquaculture, Xiamen 361021, China. ${ }^{3}$ Key Laboratory of Healthy Mariculture for the East China Sea, Ministry of Agriculture, Fisheries College, Jimei University, Xiamen 361021, China. ${ }^{4}$ Key Laboratory of Marine Biotechnology of Fujian Province, Institute of Oceanology, College of Animal Science, Fujian Agriculture and Forestry University, Fuzhou 350002, China.

Received: 20 February 2020 Accepted: 25 October 2020

Published online: 24 November 2020

\section{References}

1. Benjamin IJ, McMillan DR. Stress (heat shock) proteins: molecular chaperones in cardiovascular biology and disease. Circ Res. 1998:83(2): 117-32
2. Krone PH, Lele Z, Sass JB. Heat shock genes and the heat shock response in zebrafish embryos. Biochem Cell Biol. 1997;75(5):487-97.

3. Sreedhar AS, Csermely P. Heat shock proteins in the regulation of apoptosis: new strategies in tumor therapy: a comprehensive review. Pharmacol Ther. 2004;101(3):227-57.

4. Westerheide SD, Morimoto RI. Heat shock response modulators as therapeutic tools for diseases of protein conformation. J Biol Chem. 2005; 280(39):33097-100.

5. Åkerfelt M, Morimoto RI, Sistonen L. Heat shock factors: integrators of cell stress, development and lifespan. Nat Rev Mol Cell Biol. 2010;11(8):545.

6. Fujimoto $M$, Nakai A. The heat shock factor family and adaptation to proteotoxic stress. FEBS J. 2010;277(20):4112-25.

7. Nakai A. New aspects in the vertebrate heat shock factor system: Hsf3 and Hsf4. Cell Stress Chaperones. 1999;4(2):86.

8. Anckar J, Sistonen L. Regulation of HSF1 function in the heat stress response: implications in aging and disease. Annu Rev Biochem. 2011;80: 1089-115.

9. Hsu AL, Murphy CT, Kenyon C. Regulation of aging and age-related disease by DAF-16 and heat-shock factor. Science. 2003;300(5622):1142-5.

10. Morano KA, Santoro N, Koch KA, Thiele DJ. A trans-activation domain in yeast heat shock transcription factor is essential for cell cycle progression during stress. Mol Cell Biol. 1999;19(1):402-11.

11. Morley JF, Morimoto RI. Regulation of longevity in Caenorhabditis elegans by heat shock factor and molecular chaperones. Mol Biol Cell. 2004;15(2): 657-64.

12. Westwood JT, Clos J, Wu C. Stress-induced oligomerization and chromosomal relocalization of heat-shock factor. Nature. 1991;353(6347): 822-7.

13. Ke CH, You WW. Advances in genetics and breeding of Haliotis diversicolor [J]. J Xiamen University (Natural Science). 2011;50(002):425-30.

14. Cai XH, Huang YT, Zhang X, Wang SH, Zou ZH, Wang GD, Wang YL, Zhang $Z P$. Cloning, characterization, hypoxia and heat shock response of hypoxia inducible factor-1 (HIF-1) from the small abalone Haliotis diversicolor. Gene. 2014:534(2):256-64.

15. Huang $Y T$, Cai XH, Zou ZH, Wang SH, Wang GD, Wang YL, Zhang ZP. Molecular cloning, characterization and expression analysis of three heat shock responsive genes from Haliotis diversicolor. Fish Shellfish Immunol. 2014;36(2):590-9.

16. Zhang $X$, Huang $Y T$, Cai $X$, Zou ZH, Wang GD, Wang SH, Wang $Y L$, Zhang ZP. Identification and expression analysis of immune-related genes linked to Rel/NF-kappaB signaling pathway under stresses and bacterial challenge from the small abalone Haliotis diversicolor. Fish Shellfish Immunol. 2014;41: 200-8.

17. Wang KJ, Ren HL, Xu DD, Cai L, Yang M. Identification of the up-regulated expression genes in hemocytes of variously colored abalone (Haliotis diversicolor reeve, 1846) challenged with bacteria. Dev Comp Immunol. 2008;32(11):1326-47.

18. Huang Y. Studies on several heat shock responsive genes under thermal and hypoxic stresses in the small abalone Haliotis diversicolor. Master: Jimei University; 2014. (In Chinese)

19. Fang ZQ, Sun YL, Zhang X, Wang GD, Li YT, Wang YL, Zhang ZP. Responses of HSP70 gene to Vibrio parahaemolyticus infection and thermal stress and its transcriptional regulation analysis in Haliotis diversicolor. Molecules. 2019; 24(1):162.

20. Li YT, Zhang T, Zhang $X$, Wang GD, Wang YL, Zhang ZP. Heat shock cognate 70 gene in Haliotis diversicolor: responses to pathogen infection and environmental stresses and its transcriptional regulation analysis. Cell Stress Chaperones. 2018;23(3):335-46.

21. Zhang T, Zhang ZP, Jia XW, Wang SH, Wang GD, Wang YL. Functional analysis of the promoter in Haliotis diversicolor HSP90 gene. J Fish China. 2017:41(4):490-7 (In Chinese).

22. Richardson PG, Mitsiades CS, Laubach JP, Lonial S, Chanan-Khan AA, Anderson KC. Inhibition of heat shock protein 90 (HSP90) as a therapeutic strategy for the treatment of myeloma and other cancers. Br J Haematol. 2011;152(4):367-79.

23. Tamás MJ, Sharma SK, Ibstedt S, Jacobson T, Christen P. Heavy metals and metalloids as a cause for protein misfolding and aggregation. Biomolecules. 2014:4(1):252-67.

24. Doubrovin M, Che J, Serganova I, Moroz E, Solit D, Ageyeva L, Kochetkova T, Pillarsetti N, Finn R, Rosen N. Monitoring the induction of heat shock factor $1 /$ heat shock protein 70 expression following 17-allylamino- 
demethoxygeldanamycin treatment by positron emission tomography and optical reporter gene imaging. Mol Imaging. 2012;11:67-76.

25. Newton EM, Knauf U, Green M, Kingston RE. The regulatory domain of human heat shock factor 1 is sufficient to sense heat stress. Mol Cell Biol. 1996;16(3):839-46.

26. Rabindran SK, Giorgi G, Clos J, Wu C. Molecular cloning and expression of a human heat shock factor, HSF1. Proc Natl Acad Sci. 1991;88(16):6906-10

27. Tomanek L. The importance of physiological limits in determining biogeographical range shifts due to global climate change: the heat-shock response. Physiol Biochem Zool. 2008;81(6):709-17.

28. Zou J, Guo Y, Guettouche T, Smith DF, Voellmy R. Repression of heat shock transcription factor HSF1 activation by HSP90 (HSP90 complex) that forms a stress-sensitive complex with HSF1. Cell. 1998;94(4):471-80.

29. Morimoto RI. Regulation of the heat shock transcriptional response: cross talk between a family of heat shock factors, molecular chaperones, and negative regulators. Genes Dev. 1998;12(24):3788-96.

30. Kline MP, Morimoto RI. Repression of the heat shock factor 1 transcriptional activation domain is modulated by constitutive phosphorylation. Mol Cell Biol. 1997;17(4):2107-15.

31. Jiang Y, Loker ES, Zhang SM. In vivo and in vitro knockdown of FREP2 gene expression in the snail Biomphalaria glabrata using RNA interference. Developmental Comparative Immunol. 2006;30(10):855-66.

32. Wang GD, Li N, Zhang LL, Zhang ZP, Wang YL. IGFBP7 promotes hemocyte proliferation in small abalone Haliotis diversicolor, proved by dsRNA and cap mRNA exposure. Gene. 2015;571(1):65-70.

33. Zhang $X$, Shi JL, Sun YL, Habib YJ, Yang HP, Zhang ZP, Wang YL. Integrative transcriptome analysis and discovery of genes involving in immune response of hypoxia/thermal challenges in the small abalone Haliotis diversicolor. Fish Shellfish Immunol. 2019:84:609-26.

34. Sun YL, Zhang X, Wang YL, Day R, Yang HP, Zhang ZP. Immunity-related genes and signaling pathways under hypoxic stresses in Haliotis diversicolor: a transcriptome analysis. Sci Rep. 2019;9(1):1-15.

35. Schwarz DS, Hutvágner G, Du T, Xu Z, Aronin N, Zamore PD. Asymmetry in the assembly of the RNAi enzyme complex. Cell. 2003;115(2):199-208.

36. Tran TKA, Macfarlane GR, Kong RYC, O'Connor WA, Yu RMK. Mechanistic insights into induction of vitellogenin gene expression by estrogens in Sydney rock oysters, Saccostrea glomerata. Aquat Toxicol. 2016;174:146-58.

37. Shang X, Su J, Wan Q, Su J. CpA/CpG methylation of CiMDA5 possesses tight association with the resistance against GCRV and negatively regulates mRNA expression in grass carp, Ctenopharyngodon idella. Developmental Comparative Immunol. 2015;48(1):86-94.

38. Jones PA. Functions of DNA methylation: islands, start sites, gene bodies and beyond. Nat Rev Genet. 2012;13(7):484-92.

39. Sarda S, Das A, Vinson C, Hannenhalli S. Distal CpG islands can serve as alternative promoters to transcribe genes with silenced proximal-promoters. Genome Res. 2017:27:553-66.

40. Wang H, Li X, Bajić VB. Neural-statistical model of tata-box motifs in eukaryotes. Singapore: The Practical Bioinformatician, Institute for Infocomm Research; 2004. p. 123-56.

41. Kutach AK, Kadonaga JT. The downstream promoter element DPE appears to be as widely used as the TATA box in Drosophila core promoters. Mol Cell Biol. 2000;20(13):4754-64.

42. Pedersen AG, Baldi P, Chauvin Y, Brunak S. The biology of eukaryotic promoter prediction —a review. Comput Chem. 1999;23(3-4):191-207.

43. Zhang $X$, Huang D, Jia X, Zou Z, Wang Y, Zhang Z. Functional analysis of the promoter of the molt-inhibiting hormone (mih) gene in mud crab Scylla paramamosain. Gen Comp Endocrinol. 2018;259:131-40.

44. Kuang Q, Li J, You L, Shi C, Ji C, Guo X, Xu M, Ni Y. Identification and characterization of NF-kappaB binding sites in human miR-1908 promoter. Biomed Pharmacother. 2015;74:158-63.

45. Pei H, Hu H, Zhang X, Su C, Song X. Cloning and functional analysis of the heat-inducible promoter AtHSP70b. Chin Agri Sci Bull. 2007:4:82-6 (In Chinese),

46. Li YQ, Li J, Li HX, Wang XF, Zhu Y, Ye F, Zhang ZZ, Ren XL. Construction of an HSP 70B' promoter-driven heat-inducible vectors pHSP- shTERT and its anti-proliferative effect in breast cancer MCF-7 cells. Chin J Cancer Biotherapy. 2014;21(2):130-5.

47. Patient RK, McGhee JD. The GATA family (vertebrates and invertebrates). Curr Opin Genet Dev. 2002;12(4):416-22.

48. Reyes JC, Muro-Pastor MI, Florencio FJ. The GATA family of transcription factors in Arabidopsis and rice. Plant Physiol. 2004;134(4):1718-32.
49. Ray S, Lu Y, Kaufmann SH, Gustafson WC, Karp JE, Boldogh I, Fields AP, Brasier AR. Genomic mechanisms of p210 BCR-ABL signaling: induction of heat shock protein 70 through the GATA response element confers resistance to paclitaxel-induced apoptosis. J Biol Chem. 2004;279(34):35604-15.

50. Sun YL, Zhang X, Wang GD, Lin S, Zeng XY, Wang YL, Zhang ZP. PI3K-AKT signaling pathway is involved in hypoxia/thermal-induced immunosuppression of small abalone Haliotis diversicolor. Fish Shellfish Immunol. 2016:59:492-508.

51. You Y, Huan P, Liu B. RNAi assay in primary cells: a new method for gene function analysis in marine bivalve. Mol Biol Rep. 2012;39(8):8209-16.

52. Li N, Zhang Z, Zhang L, Wang S, Zou Z, Wang G, Wang Y. Insulin-like growth factor binding protein 7, a member of insulin-like growth factor signal pathway, involved in immune response of small abalone Haliotis diversicolor. Fish \& Shellfish Immunol. 2012;33(2):229-42.

\section{Publisher's Note}

Springer Nature remains neutral with regard to jurisdictional claims in published maps and institutional affiliations.
Ready to submit your research? Choose BMC and benefit from:

- fast, convenient online submission

- thorough peer review by experienced researchers in your field

- rapid publication on acceptance

- support for research data, including large and complex data types

- gold Open Access which fosters wider collaboration and increased citations

- maximum visibility for your research: over $100 \mathrm{M}$ website views per year

At BMC, research is always in progress.

Learn more biomedcentral.com/submissions 